\title{
Impact of Direct-Acting Antiviral Agents Treatment on Body Mass Index and Lipid Profile in Egyptian Chronic Hepatitis C Patients
}

\author{
Ayman Menesy ${ }^{1}$, Ahmed Ehab ${ }^{2, *}$, Neven Abbas ${ }^{1}$ \\ ${ }^{1}$ Internal Medicine Department, Faculty of Medicine, Mansoura University, Mansoura, Egypt; ${ }^{2}$ Gastroenterology and \\ Hepatology Department, Damietta Cardiology and gastroenterology center, Damietta, Egypt.
}

\begin{abstract}
Background: The effect of direct-acting antiviral agents (DAAs), used for the treatment of hepatitis $\mathrm{C}$ virus (HCV) on body mass index (BMI), and lipid profile parameters has a great heterogenicity and has not been well defined. This study was conducted to evaluate the impact of DAAs therapy on BMI and lipid profile in Egyptian patients with chronic HCV.

Materials and methods: This prospective cross-sectional study included 100 cases diagnosed with chronic HCV who were clinically and biochemically assessed for commencing DAAs therapy. BMI and lipid profile, as well as liver function tests, were assessed at index time and 6 months after DAAs therapy.
\end{abstract}

Results: The mean age of the included cases was 54.17 years and most of the included subjects were females (72\%). Compared to baseline parameters, there was no significant difference regard, BMI, hemoglobin levels, high-density lipoprotein (HDL), triglycerides, bilirubin, alkaline phosphatase, AFP, and creatine 6six months after the end of treatment. Furthermore, ALT, AST, and INR showed a significant decrease, however, cholesterol, low-density lipoprotein (LDL), and albumin showed a significant increase six months after the end of treatment.

Conclusions: This study demonstrates that DAAs had no impact on BMI and hemoglobin level, however, it is associated with a significant increase in LDL and cholesterol levels with no impact on HDL, triglycerides.

\section{Introduction}

Hepatitis C virus (HCV) is a globally prevalent pathogen and a leading cause of death and morbidity ${ }^{1,2}$. Chronic HCV individuals have a high risk for chronic liver disease, cirrhosis, and hepatocellular carcinoma (HCC) ${ }^{3}$.

In Egypt, an endemic area of chronic hepatitis $\mathrm{C}$, over $90 \%$ of the infections have been reported to be $\mathrm{HCV}$ genotype $4{ }^{4}$. Associations of HCV with host lipoproteins have been reported. Hepatocytes take up low-density lipoproteins (LDLs) and very-low-density lipoproteins

Keywords: Direct acting antivirals; hepatitis C; lipid profile; hemoglobin

Received: 4-3-2021; Accepted: 13-3-2021

*Corresponding author. Email:

dr.ahmed.ehab.gawish@gmail.com through LDL receptors. Antibodies to the HCV envelope may disrupt the HCV lipid-containing envelope. These antibodies could provide an efficient mode of viral entry into liver cells ${ }^{5}$.

HCV core protein localizes with apolipoprotein AII at the surface of lipid droplets, suggesting a relationship between the expression of $\mathrm{HCV}$ core protein and cellular lipid metabolism ${ }^{6}$. HCV infection or core protein expression also increases the expression of sterol regulatory element-binding protein $1 \mathrm{c}$ and its target, fatty acid synthase (FASN), which are both involved in lipid synthesis ${ }^{7}$. A previous study reported that the virus utilizes very-lowdensity lipoproteins to infect hepatocytes and several other lipid secretory mechanisms for replication with a finding of hypercholesterolemia ${ }^{8}$.

After successful treatment with interferon-based therapy, it has been shown significant increases in LDL, triglycerides, and cholesterol ${ }^{9}$. IFN-free DAA regimens for the treatment of $\mathrm{CHC}$ have demonstrated more favorable clinical outcomes, a considerable improvement in liver function, an improvement in the quality of life with an excellent safety profile ${ }^{10-12}$. However, the effects of DAAs therapy on the BMI, and lipid profile have not been investigated in detail, and the existing studies show heterogeneous results. The current study aims to assess the impact of DAAs agents on the body mass index and lipid profile in Egyptian chronic HCV patients.

\section{Materials and methods}

This prospective cross-sectional study was conducted over two years, from December 2017 to December 2019, on adult patients infected with chronic $\mathrm{HCV}$ at Mansoura University Hospitals. The study included 100 cases older than 18 years diagnosed with $\mathrm{HCV}$ infection and commenced on a DAAs treatment regimen. On the contrary, we excluded cases with the following criteria; patients with viral coinfection (HIV or hepatitis B virus), dyslipidemia, patients under lipid-lowering drugs, diabetes mellitus, hepatocellular carcinoma, previous interferon therapy, renal impairment, thyroid disorders, previous liver transplantation, and lactating females. Before participating in the study, oral consent was obtained from the included cases after an explanation of the details and complications of each procedure.

Besides, the study was approved by the local ethical committee and the Institutional Review Board of Mansoura University. All cases were subjected to detailed history 


\section{Original Article}

taking, general and abdominal examination looking for signs of chronic liver disease and liver cell failure. BMI was calculated for all cases ${ }^{13}$. Laboratory investigations included complete blood count (CBC), liver and kidney functions, alpha-fetoprotein, and thyroid function tests, along lipid profile.

For the latter one, five $\mathrm{ml}$ of fasting blood sample (8-10 h) was collected under aseptic conditions. The sample was centrifuged at $200 \mathrm{rpm}$ for $1 \mathrm{~min}$ to separate the serum that was analyzed using the commercially available working reagents (Excel Diagnostics, Hyderabad, India) for lipids.

About $0.05 \mathrm{ml}$ of a serum sample to $1 \mathrm{ml}$ of working reagent was mixed and incubated at $37^{\circ} \mathrm{C}$ and analyzed using an analyzer that works on the colorimetric principle. Cases were commenced on either of the following protocols; (1) Sofosbuvir, Daclatasvir with or with our Ribavirin, (2) Sofosbuvir and Ledipasvir, or (3) Sofosbuvir, and Semiprevir.

Six months following treatment, follow-up visits were scheduled for assessment of the effect of DAA on lipid profile and BMI. A blood sample was withdrawn for laboratory analysis of the former parameter, and that was our primary outcome. Our secondary outcome included the effect of DAAs on liver functions.

\section{Statistical analysis}

The data were tabulated and analyzed using the computer program SPSS version 20. Descriptive statistics were calculated for the data in the form of mean and standard deviation for quantitative data, or frequency and distribution for qualitative data. The student's t-test test was used to compare the mean of two groups of quantitative data whereas Fisher exact test (FET) was used to compare two groups of categorical data. A p-value $<0.05$ was considered statistically significant.

\section{Results}

Out of 100 patients included in this study, only 87 completed the study to the end and 13 patients were missed. The mean age of the included cases was 54.17 years (range, 41-68 years).

Most of the included subjects were females (72\%). Liver status by the US revealed cirrhosis in $41 \%$ of cases, while the remaining cases were non-cirrhotic. Most of the included cases were commenced on Sofosbuvir, Daclatasvir with or with our Ribavirin regimen (89\%), followed by Sofosbuvir and Semiprevir (7\%), and Sofosbuvir and Ledipasvir (4\%) (Table 1).

There was no significant difference between the twotime intervals regarding, BMI, hemoglobin levels, platelets, alkaline phosphatase, bilirubin, AFP, and creatinine. Moreover, ALT, AST, and INR showed a significant decrease 6 months after treatment, while albumin showed a significant increase as shown in (Table 2).

Regarding lipid profile changes, both HDL and triglycerides showed no significant changes 6 months after treatment ( $p=0.217$ and 0.361 respectively). Both cholesterol and LDL showed a significant increase ( $p=$ 0.011 and 0.035 respectively). Cholesterol levels increased from 165.21 up to $202.64 \mathrm{mg} / \mathrm{dl}$, while LDL increased from 98.7 up to $124.7 \mathrm{mg} / \mathrm{dl}$ after treatment (Table 3).

Table 1. Baseline demographic characteristics of the study cases.

\begin{tabular}{lcc}
\hline \multicolumn{1}{c}{ Items } & Study cases (N=100) \\
\hline Age (years) & Mean \pm SD & $54.17 \pm 5.37$ \\
\hline Sex & $28(28 \%)$ \\
\hline -Male & $72(72 \%)$ \\
\hline -Female & $(\mathrm{N} / \%)$ \\
\hline State of liver & $41(41 \%)$ \\
\hline Cirrhotic & $59(59 \%)$ \\
\hline Non-cirrhotic & $(\mathrm{N} / \%)$ \\
\hline Treatment regimen & $89(89 \%)$ \\
\hline Sofosbuvir, Daclatasvir $+/-$ Ribavirin & $4(4 \%)$ \\
\hline Sofosbuvir and Ledipasvir & $7(7 \%)$ \\
\hline Sofosbuvir and Semiprevir & \\
\hline
\end{tabular}


Table 2. Comparison of clinical and laboratory data before and 6 months after treatment.

\section{Groups}

\begin{tabular}{|c|c|c|c|}
\hline \multirow[b]{2}{*}{ Parameters } & \multicolumn{2}{|c|}{ Groups } & \multirow[b]{2}{*}{ Test of significance } \\
\hline & $\begin{array}{l}\text { Before treatment } \\
\qquad(\mathrm{N}=\mathbf{1 0 0})\end{array}$ & $\begin{array}{l}\text { After treatment } \\
\qquad(\mathrm{N}=\mathbf{8 7})\end{array}$ & \\
\hline BMI $\left(\mathrm{kg} / \mathrm{m}^{2}\right)$ & $27.59 \pm 4.19$ & $28.05 \pm 4.32$ & $P=0.129$ \\
\hline HGB (gm/dl) & $11.04 \pm 1.37$ & $10.84 \pm 1.19$ & $P=0.352$ \\
\hline Platelets $(* 10 \% / \mathrm{cmm})$ & $94.58 \pm 39.90$ & $104.41 \pm 40.69$ & $P=0.142$ \\
\hline ALT (IU/L) & $58.32 \pm 16.50$ & $29.85 \pm 11.95$ & $P<0.001$ \\
\hline AST (IU/L) & $61.86 \pm 16.69$ & $35.36 \pm 12.11$ & $P<0.001$ \\
\hline Alkaline phosphatase (IU/L) & $102.68 \pm 16.26$ & $105 \pm 13.14$ & $P=0.346$ \\
\hline Albumin $(g / d l)$ & $3.16 \pm 0.36$ & $3.38 \pm 0.34$ & $\mathrm{P}<0.001$ \\
\hline INR & $1.29 \pm 0.18$ & $1.22 \pm 0.14$ & $P=0.012$ \\
\hline Total bilirubin (mg/dl) & $1.89 \pm 0.53$ & $1.06 \pm 0.36$ & $P=0.087$ \\
\hline Direct bilirubin (mg/dl) & $0.94 \pm 0.44$ & $0.72 \pm 0.18$ & $P=0.094$ \\
\hline AFP (ng/ml) & $5.50 \pm 2.85$ & $5.61 \pm 6.86$ & $\mathrm{P}=0.893$ \\
\hline Creatinine (mg/dl) & $0.96 \pm 0.22$ & $0.94 \pm 0.20$ & $P=0.669$ \\
\hline
\end{tabular}

BMI, body mass index; HGB, hemoglobin; ALT, alanine aminotransferase; AST, aspartate transaminase; INR, international normalized ratio; $\boldsymbol{A F P}$, alpha-fetoprotein.

Table 3. Comparison of lipid profile parameters before and after treatment.

\section{Groups}

\begin{tabular}{lccc} 
& $\begin{array}{c}\text { Before treatment } \\
(\mathbf{N}=\mathbf{1 0 0})\end{array}$ & $\begin{array}{c}\text { After treatment } \\
(\mathbf{N}=\mathbf{8 7})\end{array}$ & Test of significance \\
\cline { 1 - 2 } Parameters & $165.21 \pm 33.19$ & $202.64 \pm 37.5$ & $\mathrm{P}=0.011$ \\
\hline $\mathbf{m g} / \mathbf{d l})$ & $55.34 \pm 16.52$ & $61.47 \pm 21.03$ & $\mathrm{P}=0.217$ \\
\hline $\mathbf{l})$ & $98.7 \pm 28.1$ & $124.7 \pm 34.8$ & $\mathrm{P}=0.035$ \\
\hline
\end{tabular}

HDL, high-density lipoprotein; $\mathbf{L D L}$, low-density lipoprotein; TGs: triglycerides.

\section{Discussion}

The previous study in the era of IFN treatment of chronic HCV demonstrated a post-SVR increase in increasing body mass index in nondiabetic CHC patients ${ }^{14,15}$. However, the effect of direct-acting antiviral agents used for the treatment of hepatitis $\mathrm{C}$ virus on body mass index and lipid profile parameters has a great heterogenicity and has not been well defined in the era of direct-acting antiviral agents. In the current study, there was no significant difference between the BMI of the included cases before and after treatment with DAA. It had mean values of 27.59 and $28.05 \mathrm{~kg} / \mathrm{m}^{2}$ before and after treatment respectively. In line with our findings, Gitto et al. found a nonsignificant difference in
BMI following DAAs therapy in patients with chronic HCV infection. The BMI changed from $26.2 \mathrm{~kg} / \mathrm{m} 2$ before therapy to $26.0 \mathrm{~kg} / / \mathrm{m} 2$ at the 24 -week follow-up ${ }^{16,17}$. In contrast to our findings, another study reported a significant increase in BMI after DAAs treatment ${ }^{18}$. The reason for such an increase in BMI with DAA treatment is unclear. It may be related to the improvement of psychological state, i.e., an increase in appetite or taste sensation with a clearance of $\mathrm{HCV}^{19}$. Another explanation is that successful treatment is associated with a reduction in inflammatory cytokines and TNF-alpha, both of which act as cofactors in weight loss ${ }^{20,21}$. In our study, no significant difference was noted regarding pre-and post-treatment hemoglobin levels, 
which had mean values of 11.4 and $10.84 \mathrm{gm} / \mathrm{dl}$ before and after treatment respectively. Conversely, El Sagheer et al. reported a significant decrease in hemoglobin level from 13.6 before treatment down to $12.7 \mathrm{~g} / \mathrm{dl}$ after ${ }^{22}$. Another study reported a significant change in hemoglobin level after DAAs treatment $(\mathrm{p}<0.001)$. It had mean values of 13.19 and $12.57 \mathrm{gm} / \mathrm{dl}$ before and after treatment respectively ${ }^{18}$. Additionally, Alhaddad and his coworkers also reported a significant change in hemoglobin level after DAA therapy. It decreased from $12.28 \mathrm{gm} / \mathrm{dl}$ down to 10.09 $\mathrm{gm} / \mathrm{dl}$ after treatment ${ }^{23}$. In the current study, no significant changes were detected regarding platelet count before and after treatment. Likewise, Shousha and his associates also reported no significant difference regarding platelet count ${ }^{18}$. Also, El Sagheer et al. reported that platelets had mean values of 196.09 and 194.6 before and after treatment respectively, with no significant difference between the two levels $(p=0.7)^{22}$.

The current study demonstrated a significant elevation of albumin levels after DAAs treatment, as it increased from $3.16 \mathrm{~g} / \mathrm{dl}$ before therapy up to $3.38 \mathrm{gm} / \mathrm{dl}$ after therapy. In line with our findings, another study reported a significant increase in albumin levels after DAAs therapy. it increased from $3.6 \mathrm{~g} / \mathrm{dl}$ before treatment up to $4.02 \mathrm{~g} / \mathrm{dl}$ after treatment 23. On the contrary, Abdulhameed et al. reported a significant decrease in albumin levels after DAA therapy, however, that significance was not clinically significant as both of the pre-treatment and post-treatment values were within the normal range. They had mean values of 4.2 and $4.1 \mathrm{gm} / \mathrm{l}$ before and after treatment respectively ${ }^{24}$. Our study also revealed a significant decrease in the hepatic transaminase levels after DAAs therapy. This could be explained by regression of hepatic inflammation after achieving SVR. In line with our findings, other studies reported that there was a significant decrease in the hepatic transaminases post-SVR following DAAs ${ }^{18,22,23}$.

Our findings revealed that INR showed a significant improvement after DAAs treatment as it decreased from 1.29 down to $1.22(\mathrm{p}=0.012)$. This could be explained by the improvement of hepatic function after therapy including coagulation factor synthesis. Contrarily, Shousha et al. negated any significant change in INR after DAAs therapy 18. In accordance with previous studies, we found a nonsignificant change in serum bilirubin, alkaline phosphatase and alpha fetoprotein levels post-DAAs-SVR 18,22-25. On the other hand, Alhaddad and his associates reported a significant decrease in serum bilirubin levels after DAA combined with ribavirin treatment $(\mathrm{p}<0.001)$. It decreased from $1.5 \mathrm{mg} / \mathrm{dl}$ before treatment down to 0.9 $\mathrm{mg} / \mathrm{dl}$ after treatment ${ }^{23}$.

As regarding lipid profile changes in the current study, total cholesterol levels showed a significant increase with DAAs therapy. This effect may be attributed to the reversal of the impact of HCV replication on hepatic lipid metabolism ${ }^{26}$. In accordance with our result many studies demonstrated increasing the levels of total cholesterol after DAA therapy $16,17,22,27$. On the other hand, another study negated any significant changes regarding total cholesterol level after DAA therapy. It had mean values of 147.2 and $174.6 \mathrm{mg} / \mathrm{dl}$ before and after treatment respectively ${ }^{24}$. In the current study, there was no significant changes in serum triglyceride (TG) post-DAAs-SVR. In line with our finding, previous studies negated any significant changes in TG levels after DAA therapy ${ }^{24,25,28}$. However, in contrast to our result, El Sagheer and his colleagues reported that DAAs therapy led to a significant decrease in TG levels, which decreased from $100.5 \mathrm{mg} / \mathrm{dl}$ before therapy down to 81.7 $\mathrm{mg} / \mathrm{dl}$ after it $(\mathrm{p}=0.001)^{22}$. On the contrary, Kamal et al. reported that DAAs were associated with a significant rise in TG levels ${ }^{17}$. In our study, we did not witness any significant changes regarding HDL levels post-treatment. In agreement with our results, Morales et al. also reported no significant changes regarding HDL levels (pre $52.8 \pm 18.3$ $\mathrm{mg} / \mathrm{dL}$, post $51.4 \pm 18.5 \mathrm{mg} / \mathrm{dL}-\mathrm{P}=0.699)^{28}$. Russo et al. also reported no significant changes regarding HDL levels which changed from $58 \mathrm{mg} / \mathrm{dl}$ before treatment to $55 \mathrm{mg} / \mathrm{dl}$ following it $(p>0.05)^{25}$. Gitto et al. reported the previous findings ${ }^{16}$. In contrast, another study reported significant changes in HDL levels after DAA therapy ( $\mathrm{p}=0.038)$, as it had mean values of 37.4 and $39.8 \mathrm{mg} / \mathrm{dl}$ before and after treatment respectively ${ }^{24}$. El Sagheer et al. and Kamal et al. reported the same changes ${ }^{17,22}$. In the current study, LDL showed a significant increase after DAA treatment compared with baseline. It increased from $98.7 \mathrm{mg} / \mathrm{dl}$ before treatment up to $124.7 \mathrm{mg} / \mathrm{dl}$ after treatment. The above findings are supported by the findings of Ichikawa $\mathrm{T}$ et al., who found that in patients achieving an SVR after DAAs treatment, the LDL increased a year after starting treatment ${ }^{29}$. Karthik, et al. suggested that the rapid suppression of HCV core proteins due to DAAs may lead to a deregulation of host lipid metabolism that manifests as a decrease of lipid droplet production in HCV-infected liver cells and a large rebound of circulating LDL ${ }^{30}$. Another study reported a significant difference regarding LDL levels, which increased from $87.8 \mathrm{mg} / \mathrm{dl}$ before DAAs therapy up to $111.0 \mathrm{mg} / \mathrm{dl}$ after treatment ${ }^{24}$. Furthermore, Gitto et al. reported also a significant increase in LDL levels from $80 \mathrm{mg} / \mathrm{dl}$ before treatment up to $102 \mathrm{mg} / \mathrm{dl}$ after treatment $(p<0.001)^{16}$. Based on the above data, it appears that there is a great heterogenicity of data regarding the effect of DAAs therapy on lipid profile. This matter needs to be elucidated in a meta-analysis or by a global study including population from different countries. Also, molecular studies handling this perspective should be encouraged. The lack of pathologic data along with transient elastography assessment represents some of the limitations of the current study. Other limitations are the relatively small sample size that was collected from one tertiary medical center. Furthermore, there was no enough number of cases commenced on each regimen to compare the effect of each DAA regimens on the studied parameters. Hence, the upcoming studies should include more cases from different centers to cover these drawbacks.

\section{Conclusion}

This study demonstrates that DAAs had no impact on $\mathrm{BMI}$ and hemoglobin level, however, it is associated with a significant increase in LDL and cholesterol levels with no impact on HDL, triglycerides. Furthermore, DAAs therapy 
for $\mathrm{HCV}$ is associated with a significant improvement in liver function.

\section{References}

1. Ward, J. W., Hinman, A. R., and Alter, H. J. (2020). Time for the elimination of hepatitis $\mathrm{C}$ virus as a global health threat. The liver: biology and pathobiology, 935952.

2. Bajis, S., Applegate, T. L., Grebely, J., et al. (2020). Novel Hepatitis C Virus (HCV) Diagnosis and Treatment Delivery Systems: Facilitating HCV Elimination by Thinking outside the Clinic. The Journal of infectious diseases, 222(Supplement_9), S758-S772.

3. Yan, P., Pang, P., Hu, X., et al. (2021). Specific MiRNAs in naïve $T$ cells associated with Hepatitis C Virus-induced Hepatocellular Carcinoma. Journal of Cancer, 12(1), 1.

4. El-Akel, W., El-Sayed, M. H., El Kassas, M., et al. (2017). National treatment programme of hepatitis $\mathrm{C}$ in Egypt: hepatitis $\mathrm{C}$ virus model of care. Journal of viral hepatitis, 24(4), 262-267.

5. Kanda, T., and Moriyama, M. (2017). Direct-acting antiviral agents against hepatitis $\mathrm{C}$ virus and lipid metabolism. World journal of gastroenterology, 23(31), 5645.

6. Grassi, G., Di Caprio, G., Fimia, G. M., et al. (2016). Hepatitis C virus relies on lipoproteins for its life cycle. World journal of gastroenterology, 22(6), 1953.

7. Bose, S. K., Kim, H., Meyer, K., et al. (2014). Forkhead box transcription factor regulation and lipid accumulation by hepatitis $\mathrm{C}$ virus. Journal of virology, 88(8), 4195-4203.

8. Li, C., Lu, L., Qi, Z., et al. (2020). Transcriptome and miRNome Analysis Provide New Insight into Host Lipid Accumulation, Innate Immunity, and Viral Persistence in Hepatitis C Virus Infection in vitro. Frontiers in Microbiology, 11.

9. Kuo, Y. H., Chuang, T. W., Hung, C. H., et al. (2011). Reversal of hypolipidemia in chronic hepatitis $\mathrm{C}$ patients after successful antiviral therapy. Journal of the Formosan Medical Association, 110(6), 363-371.

10. Kamal, S. M., and Nasser, I. A. (2008). Hepatitis C genotype 4: What we know and what we don't yet know. Hepatology, 47(4), 1371-1383.

11. Dina, E., Nasser, M., Sarah, E., et al. (2020). Pretreatment serum alpha fetoprotein and its relation to sustained virologic response in patients with chronic $\mathrm{HCV}$ infection treated with direct-acting antiviral therapy. Medical Journal of Viral Hepatitis, 4(2), 6973.

12. Dina, E., Nasser, M., Sarah, E., et al. (2020). Pretreatment serum alpha fetoprotein and its relation to sustained virologic response in patients with chronic $\mathrm{HCV}$ infection treated with direct-acting antiviral therapy. Medical Journal of Viral Hepatitis, 4(2), 6973.
13. Ogden, C. L., Carroll, M. D., Curtin, L. R., et al. (2006). Prevalence of overweight and obesity in the United States, 1999-2004. Jama, 295(13), 1549-1555.

14. Mousa, N., Abdel-Razik, A., Farag, R., et al. (2017). Do we need to screen for de-novo diabetes mellitus in chronic hepatitis $\mathrm{C}$ patients after a sustained virological response? European journal of gastroenterology \& hepatology, 29(9), 1004-1009.

15. Rodriguez-Torres, M., Sulkowski, M. S., Chung, R. T., et al. (2010). Factors associated with rapid and early virologic response to peginterferon alfa-2a/ribavirin treatment in $\mathrm{HCV}$ genotype 1 patients representative of the general chronic hepatitis C population. Journal of viral hepatitis, 17(2), 139-147.

16. Gitto, S., Cicero, A. F., Loggi, E., et al. (2018). Worsening of serum lipid profile after direct acting antiviral treatment. Annals of hepatology, 17(1), 64-75.

17. Kamal, E. M., El-Sayed, A. M., and Mohammed, M. A. Effect of Direct-Acting Antivirals on Lipid and Glucose Metabolism in Chronic HCV Patients.

18. Shousha, H. I., Abdelaziz, R. A., Azab, S. M., Khairy, et al. (2018). Effect of treatment with direct acting antivirals on body mass index and hepatic steatosis in chronic hepatitis C. Journal of medical virology, 90(6), 1099-1105.

19. Musialik, J., Suchecka, W., Klimacka-Nawrot, E., et al. (2012). Taste and appetite disorders of chronic hepatitis $\mathrm{C}$ patients. European journal of gastroenterology \& hepatology, 24(12), 1400-1405.

20. Villani, R., Facciorusso, A., Bellanti, F., et al. (2016). DAAs rapidly reduce inflammation but increase serum VEGF level: a rationale for tumor risk during anti-HCV treatment. PloS one, 11(12), e0167934.

21. Kayacan, O., Karnak, D., Beder, S., et al. (2006). Impact of TNF- $\alpha$ and IL- 6 levels on development of cachexia in newly diagnosed NSCLC patients. American journal of clinical oncology, 29(4), 328-335.

22. El Sagheer, G., Soliman, E., Ahmad, A., et al. (2018). Study of changes in lipid profile and insulin resistance in Egyptian patients with chronic hepatitis $C$ genotype 4 in the era of DAAs. Libyan Journal of Medicine, 13(1).

23. Alhaddad, O., Wahb, A., Sabry, A., et al. (2020). Role of Ribavirin in the Era of Direct-Acting Antiviral Therapies of Chronic Hepatitis C. Expert review of anti-infective therapy, 18(8), 817-822.

24. Abdulhameed, N. I., Aleem, M. S. A., Shatat, M., et al. (2020). changes in serum lipid profiles and apolipoprotein levels during therapy with daas in egyptian patients infected with hepatitis c virus genotype 4. Journal of Critical Reviews, 7(10), 31743178.

25. Russo, F. P., Zanetto, A., Gambato, M., et al. (2020). Hepatitis $\mathrm{C}$ virus eradication with direct-acting antiviral improves insulin resistance. Journal of viral hepatitis, 27(2), 188-194. 
26. Mauss, S., Berger, F., Wehmeyer, M. H., et al. (2017). Short communication Effect of antiviral therapy for HCV on lipid levels. Antiviral therapy, 21, 81-88.

27. Doyle, M. A., Galanakis, C., Mulvihill, E., et al. (2019). Hepatitis $\mathrm{C}$ direct acting antivirals and ribavirin modify lipid but not glucose parameters. Cells, 8(3), 252.

28. Morales, A. L., Junga, Z., Singla, M. B., et al. (2016). Hepatitis C eradication with sofosbuvir leads to significant metabolic changes. World journal of hepatology, 8(35), 1557.

29. Ichikawa, T., Miyaaki, H., Miuma, S., et al. (2019). Carotid intima-media thickness and small dense lowdensity lipoprotein cholesterol increase after one year of treatment with direct-acting antivirals in patients with hepatitis $\mathrm{C}$ virus infection. Internal Medicine, 1514-18.

30. Karthik, L., Kumar, G., Keswani, T., et al. (2014). Protease inhibitors from marine actinobacteria as a potential source for antimalarial compound. PloS one, 9(3), e90972. 